\title{
Idiopathic Pulmonary Fibrosis Update on Genetic Discoveries
}

\author{
Christine Kim Garcia ${ }^{1}$ \\ ${ }^{1}$ McDermott Center for Human Genetics, University of Texas Southwestern Medical Center, Dallas, Texas
}

\begin{abstract}
Idiopathic pulmonary fibrosis (IPF) is a progressive fibrotic disease of the lungs that increases in prevalence with advanced age. Recent evidence indicates that mutations in genes of two different biologic pathways lead to the common phenotype of familial pulmonary fibrosis (FPF) and sporadic IPF. Mutations in the genes encoding the lung surfactant proteins $\mathrm{C}$ and $\mathrm{A} 2$ (SFTPC and SFTPA2, respectively) cause increased endoplasmic reticulum stress in type II alveolar epithelial cells. Mutations in the genes encoding telomerase (TERT and TERC) cause IPF through shortening of telomere lengths and probable exhaustion of lung stem cells. All of the mutations are individually rare, but, collectively, TERT mutations are the most common genetic defect found in FPF. The overall penetrance of pulmonary fibrosis in TERT mutation carriers is $40 \%$ in subjects with a mean age of 51 years. Penetrance increases with advanced age, is greater in males than in females, and is positively associated with fibrogenic environmental exposures. Short telomere lengths are found in patients with FPF and sporadic IPF without mutations in telomerase, suggesting that the biologic pathway of telomerase dysfunction provides a biologic explanation for the age-related prevalence of IPF. The molecular data of two seemingly unrelated biologic pathways-alveolar epithelial endoplasmic reticulum stress and telomerase dysfunction-are beginning to elucidate the pathogenesis of IPF. These results have potentially predictive and therapeutic value.
\end{abstract}

Keywords: genetics; idiopathic pulmonary fibrosis; surfactant proteins; telomerase; telomere length

The interstitial lung diseases (ILDs) are a heterogeneous collection of over 100 different diseases, all characterized by pulmonary fibrosis. Each disorder is distinguished by clinical, radiographic, and pathologic characteristics. Idiopathic pulmonary fibrosis (IPF) is the prototypic ILD. IPF represents an extreme in the spectrum of the different ILDs in that it is the most deadly. It is progressive and fatal with a median life expectancy of 2.5-3.5 years after diagnosis (1). Over 30,000 new cases of IPF are diagnosed each year in the United States, with epidemiologic data suggesting that the incidence and mortality is rising, especially in white and Hispanic populations $(2,3)$. Although once considered an orphan disease, the mortality rate from IPF has now surpassed those from a variety of different malignancies, including acute myelogeneous leukemia, multiple myeloma, and bladder cancer (3). One of the hallmark characteristics of IPF is its striking increase with age (4). The prevalence of IPF is approximately 100-fold higher for those over 75 years of age than those under 35 years of age. The disease preferentially affects males and smokers.

(Received in original form August 4, 2010; accepted in final form January 3, 2011) Supported, in whole or in part, by National Institutes of Health grant HL093096, the Doris Duke Charitable Foundation, and the American Heart Association.

Correspondence and requests for reprints should be addressed to Christine Kim Garcia, M.D., Ph.D., McDermott Center for Human Genetics, University of Texas Southwestern Medical Center, 5323 Harry Hines Blvd, Dallas, TX 75390-8591. E-mail: christine.garcia@utsouthwestern.edu

Proc Am Thorac Soc Vol 8. pp 158-162, 2011

DOI: $10.1513 /$ pats.201008-056MS

Internet address: www.atsjournals.org
Until recently, there was no known cause of IPF. Dissecting the genetic underpinnings of this disease has led to advances in our understanding of IPF. Marshall and colleagues (5) published the first large collection of familial IPF kindreds by systematically surveying pulmonary physicians in the United Kingdom. They identified 57 cases from 21 families and estimated that $0.5-2.2 \%$ of cases were familial, with an overall prevalence of 1.34 cases per million. They found that the clinical presentation of familial IPF appeared to be indistinguishable from the more common sporadic form of the disease (patients with IPF with no positive family history), except for an earlier age of onset. The mean age of diagnosis for familial IPF was 55 years, compared with 68 years for the sporadic cases. Subsequent studies found higher frequencies of the familial subtype in lung transplantation centers $(6,7)$. Up to $50 \%$ and $36 \%$ of familial cases reported a positive smoking history or exposure to a fibrogenic agent, respectively, suggesting a positive geneticenvironmental interaction (5). The largest cohort of familial IPF was reported in 2005 (8). Many of the pedigrees showed vertical transmission consistent with a genetic pattern of autosomal dominant inheritance with reduced penetrance. Interestingly, $45 \%$ of the pedigrees showed phenotypic heterogeneity, with different idiopathic interstitial pneumonia (IIP) pathologies observed in different affected individuals within the same family (8). This suggested that the underlying genetic factor(s) caused an increased predisposition for pulmonary fibrosis, and not a predisposition for one particular pathologic subtype. The phenotype of some of these kindreds is more accurately described as familial pulmonary fibrosis (FPF) rather than familial IPF.

\section{MUTATIONS IN THE GENE ENCODING SURFACTANT PROTEIN C}

The gene encoding surfactant protein (SP)-C was tested as a candidate gene in one large kindred by Thomas and colleagues (9). This family spanned 5 generations and contained 11 individuals with pulmonary fibrosis (including four with biopsyproved usual interstitial pneumonia [UIP]) and 3 affected with biopsy-proved nonspecific interstitial pneumonia (NSIP). The age of diagnosis of the affected individuals ranged from 4 months to 57 years. Sequencing the coding regions of the gene revealed a novel nonsynonymous variant that was predicted to change the leucine at position 188 to a glutamine (L188Q). This mutation was present in all affected individuals, but was not found in four unaffected family members or 88 control chromosomes. Multipronged evidence suggested that this variant was indeed the culprit mutation. There was abnormal staining of SP-C in the lung tissue of the affected individuals, there was an absence of well formed lamellar bodies in the alveolar type II cells, and mouse lung epithelial cells transfected with the mutant SP-C construct showed increased cytotoxicity compared with those transfected with the wild-type gene.

Previously, Nogee and colleagues (10) described a small nuclear family consisting of an affected mother with desquamative interstitial pneumonitis and an affected daughter with NSIP who both inherited a heterozygous mutation in the SP-C 
gene that resulted in the skipping of exon 4 and the deletion of the terminal 37 amino acids. There was no normal SP-C in the lung tissue of the affected child suggesting a dominant negative effect of the mutant protein. Later reports demonstrated that the molecular mechanism of this mutation involved its deleterious effect on the cellular trafficking of normal SP-C protein and its induction of the unfolded protein response and endoplasmic reticulum (ER)-associated degradation (ERAD) pathway $(11,12)$.

The expression of the SP-C gene is restricted to alveolar type II epithelial cells. The SP-C mutations suggest that injury to these cells and/or the decreased SP-C expression underlies the development of various ILDs in mutation carriers. Mutations in the SP-C are rare in individuals without a positive family history of pulmonary fibrosis. In one study, mutations in SP-C were found in less than $1 \%$ of patients with sporadic UIP or NSIP (13).

\section{MUTATIONS IN THE GENE ENCODING SP-A}

Other large kindreds with familial pulmonary fibrosis have been extensively characterized. In one, 11 members were diagnosed with pulmonary fibrosis and 5 died before 50 years of age (14). One unique phenotype of this kindred is adenocarcinoma of the lung with features of bronchoalveolar cell carcinoma cosegregated with ILD, which was found in four individuals; three additional individuals had lung cancer without an ILD. Wholegenome linkage of 29 family members was performed using a single nucleotide polymorphism (SNP) panel of more than 6,000 markers. Individuals with pulmonary fibrosis and/or lung cancer were classified as "affecteds," and all others were assigned an unknown affectation status. The data analysis revealed a single significant peak on chromosome 10 at 10q22 that spanned $15.7 \mathrm{Mb}$ and encompassed 118 annotated genes. The three top candidate genes found within the linked region were those encoding SP-A1, the closely related isoform, SP-A2, and SP-D. These three genes were considered candidates because of their lung-specific expression profile, their key roles in the alveoli in the distal lung, and the precedence of the discovery of an SP-C mutation in an FPF kindred.

Sequencing these three genes led to the discovery of a novel missense variant in the gene encoding SP-A2 that predicted a change of a glycine at position 231 to a valine (G231V) (14). The coding regions of these three genes were sequenced in additional FPF probands, and a second kindred was discovered with a rare variant predicted to change the phenylalanine at position 198 to a serine. Both G231V and F198S variants are predicted to change the amino acid sequence at highly conserved positions within the carbohydrate recognition domain of the SP-A2 protein. Modeling these two changes on the known crystal structure of the rat SP-A carbohydrate recognition domain predicts instability of the translated protein.

Distinguishing the biochemical effects of the G231V and F198S variants is important for understanding their pathogenesis. The biochemical properties of these two variants have been compared with common SP-A2 variants found in the normal population or rare variants of unknown pathogenicity $(14,15)$. Each variant was constructed on the backbone of the wild-type SP-A2 sequence with an engineered N-terminal tag for identification by Western blotting or immunoprecipitation. Only the mutagenic F198S and G231V variants are not secreted into the tissue culture media from transfected A549 or human type II alveolar epithelial cells. All other variants tested, including T9N, V50L, A91P, Q223K, and the rare variant, L12W, which does not segregate with pulmonary fibrosis in the kindred in which it was discovered, are readily made in the cell lysate, post-translationally modified and secreted into the culture media. In comparison to the wild-type or other variants, the G231V and F198S mutant proteins are retained in the ER, fail to make higher-order oligomers, are found in a detergent-insoluble fraction in A549 cells, demonstrate greater sensitivity to digestion by $\alpha$-chymotrypsin, and increase cellular markers of ER stress.

As the SP-A2 mutations create a protein that is not readily secreted out of the transfected cells, the mechanism of disease may, in part, relate to a decreased concentration of mature SP-A in the alveolar space. Bronchoalveolar lavage samples were collected from different subjects from the G231V kindred. Western blots of the bronchoalveolar lavage fluid demonstrate that the related family members had roughly equivalent amounts of total SP-A, -B, and -D, regardless of whether or not they had inherited the G231V mutation (15). The mechanism of this SPA2 mutation involves increased ER stress of the type II alveolar epithelial cells without a decrease of total secreted SP-A. This finding differentiates the SP-A2 mutations from the SP-C mutations, which cause both increased ER stress and decreased expression of SP-C in the lung.

\section{MUTATIONS IN THE GENES ENCODING TELOMERASE (TERT AND TERC)}

Telomerase is a multimeric ribonucleoprotein enzyme that catalyzes the addition of repetitive DNA sequence to the telomeres, or specialized structures at the ends of chromosomes $(16,17)$. The enzymatic action of telomerase solves the end-replication problem by counteracting the progressive shortening of the chromosome that occurs with each cell division. In humans, the protein component of telomerase (encoded by the TERT gene) is expressed only in germ cells, cells with proliferative potential, and immortalized cancer cells (18-20). Restricted expression of telomerase in humans is evidenced by the progressive shortening of telomere lengths with age (21). The human disease, dyskeratosis congenita (DC), is characterized by short telomeres and inherited telomerase dysfunction (22). Other diseases, including pulmonary fibrosis, bone marrow failure syndromes, and liver disorders, are "telomeropathies" in that they are caused by germ line mutations in the genes encoding telomerase, and are characterized by short telomere lengths.

The first clue that telomerase dysfunction is related to pulmonary fibrosis came in 2005 when Armanios and colleagues (23) characterized one kindred with DC with a heterozygous missense mutation in the gene encoding the protein component of telomerase (TERT). Four of the seven mutation carriers, ranging in age from 21 to 63 years, carried a diagnosis of IPF. An independent cohort of FPF was sequenced for mutations in the telomerase genes, and 6 of 73 kindreds were found with a loss of function mutation in TERT (24). An additional pedigree was described with a mutation in the gene encoding the RNA component of telomerase, TERC (24). Using an unbiased genetic approach, we performed a whole-genome linkage scan of two large kindreds with FPF (25). Individuals with IPF or progressive end-stage pulmonary fibrosis were considered as "affecteds." We found evidence of linkage to the short arm of chromosome 5 in a small region that contained the TERT gene. Given the known association between the TERT mutation and FPF in the DC family, the TERT gene was sequenced. Two heterozygous mutations, a missense $\mathrm{R} 865 \mathrm{H}$ mutation and a V747fs frameshift mutation, were discovered in the probands of these two kindreds.

We have sequenced the coding regions of TERT and TERC for the probands of 106 unrelated kindreds with adult-onset FPF, and have found $19(18 \%)$ with heterozygous TERT mutations and $1(\sim 1 \%)$ with a heterozygous TERC mutation (26). Similarly, we have found two cases of heterozygous TERT 
mutations (3\%) in a group of unrelated patients with IIPs and no family history of lung disease (27). The higher prevalence of telomerase mutations in the familial cases reflects the effect of these mutations in causing the pulmonary fibrosis phenotype. Their presence in the sporadic cases indicates that penetrance is incomplete. Each of these TERT mutations is individually rare; none have been found in a sequenced reference control cohort (28). However, collectively, TERT mutations are the most common genetic defect found in patients with IPF.

The mutations span the entire length of the gene with a higher concentration of mutations found in the reverse-transcriptase and $\mathrm{C}$-terminal regions than in the $\mathrm{N}$-terminal region. They are all loss-of-function frameshift, splice site, or missense mutations, with most demonstrating decreased in vitro telomerase activity. Cotranslation of different ratios of the V747fs and wildtype TERT protein did not negatively affect the activity of the wild-type protein, suggesting a mechanism of haploinsufficiency (25). All mutations segregate with pulmonary fibrosis in the kindreds in which they are found. All are associated with short telomere lengths; all subjects with these mutations have telomere lengths above the 50th percentile, and most have telomere lengths below the 10th percentile. The majority of mutations are private for the family in which it was found, with a few exceptions. The V144M and the R951W mutations have been found in unrelated kindreds (26).

\section{PHENOTYPE OF TERT MUTATION CARRIERS}

We have expanded the FPF kindreds with identified TERT mutations and have identified 134 carriers of heterozygous mutations, ranging in age from 5 to 88 (26). The penetrance of pulmonary fibrosis is related to age, varying from zero for individuals under 40 years to approximately $60 \%$ for men and $50 \%$ for women 60 years of age or older. Overall, $40 \%$ of TERT mutation carriers with a mean age of 51 years have self-reported pulmonary fibrosis. The majority of TERT mutation carriers with pulmonary fibrosis carry a diagnosis of IPF, but some do not fit the narrow diagnostic criteria for this disease. Threefourths of 39 patients had a radiographic pattern that is typical of UIP, and 13 had a pattern consistent with UIP with the absence of honeycombing. Five patients (13\%) had a radiographic pattern that is atypical of IPF, with either predominant reticulations in the upper- or mid-lung zones, or with fibrosis concentrated along the bronchi. Review of the surgical lung biopsies demonstrated that 25 of 29 cases ( $86 \%$ ) are compatible with a pathologic diagnosis of UIP. In 10 cases (35\%), chronic inflammation was increased compared with that usually seen in typical UIP. Five cases (17\%) had scattered histiocytes and/or small, loosely formed granulomas within the interstitium, which are not usually seen in typical UIP. Superimposed areas of acute lung injury, including bronchiolitis obliterans with organizing pneumonia (BOOP) and diffuse alveolar damage (DAD), were seen in four cases. The mean life expectancy of TERT mutation carriers with pulmonary fibrosis was 3 years from the time of diagnosis, regardless of the ILD diagnosis. This clinical outcome of progressive pulmonary fibrosis due to TERT mutations mirrors the clinical course of IPF.

Overall, the TERT mutation carriers died at an earlier age (26). The average age at death for males was 57.7 years. For female mutation carriers, the average age of death was 66.6 years. This is in comparison with the average life expectancy in the United States in 2006 of 75.1 and 80.2 years for men and women, respectively. The TERT mutation carriers carry a number of diagnoses besides pulmonary fibrosis that are also seen in DC patients. However, these diseases are usually milder in the TERT families than in patients with DC. For example, $13 \%$ of patients with TERT mutations identified from FPF kindreds have a mild anemia, and $3 \%$ of mutation carriers have aplastic anemia or myelodysplastic syndrome. In contrast, greater than $85 \%$ of patients with DC have aplastic anemia (29).

What might determine the spectrum of organ dysfunction in TERT mutation carriers? We have collected environmental exposure data from all study participants. We find that over $95 \%$ of the mutation carriers with pulmonary fibrosis over 40 years of age have a past history of cigarette smoking or a history of an exposure to a fibrogenic agent, such as a medication, bird antigens, asbestos, or metal or dust particulates (26). There is a significant association between environmental lung injury, smoking and/or fibrogenic exposures, with the development of pulmonary fibrosis in TERT mutation carriers.

\section{TELOMERE LENGTHS OF PATIENTS WITH IPF}

How do mutations in telomerase cause disease? Most of the evidence suggests that telomere shortening is the key to the pathogenesis of these mutations in humans (30). Inherited TERT mutations cause a shorter inherited telomere length set point in all somatic cells. Injuries to the lung from smoking, pneumonia, or other environmental insults may be inciting events. In response, there is hyperplasia and proliferation of the alveolar epithelial cells; these alveolar cells secrete a plethora of profibrotic cytokines at the leading edge of an exuberant fibrotic response (31). Quiescent progenitor cells with replicative potential are likely recruited to regenerate the pool of injured alveolar epithelial cells. The proliferation of these stem-like cells leads to further telomere shortening, followed by senescence after telomeres reach a critically short length. The premature exhaustion of the lung stem cells over time, the premature
Familial Pulmonary Fibrosis

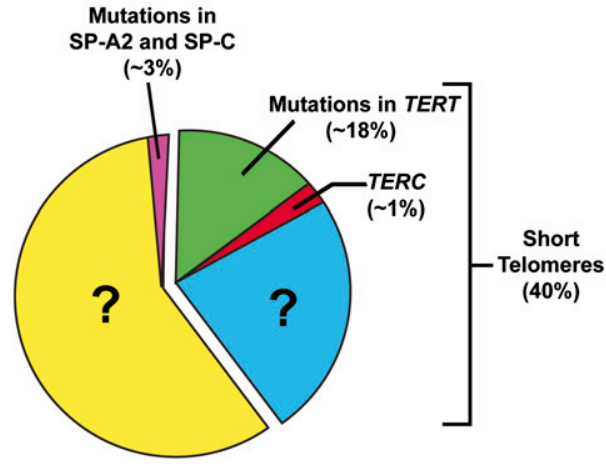

\section{Sporadic Pulmonary Fibrosis}

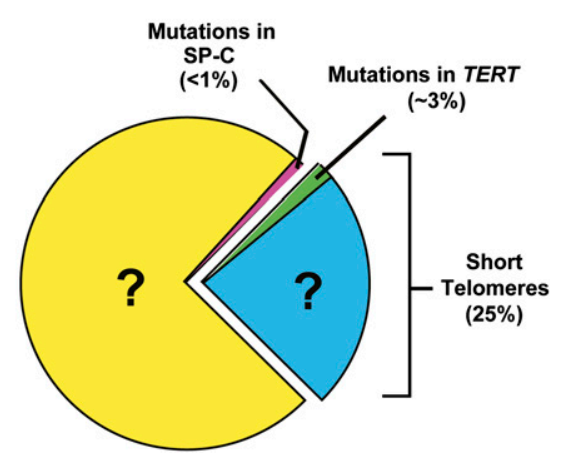

Figure 1. Estimated frequencies of mutations in genes in patients with adult-onset familial and sporadic pulmonary fibrosis. Mutation frequencies of genes encoding surfactant protein C (SFTPC), surfactant protein A2 (SFTPA2), and telomerase (TERT and TERC) as well as the percentage of patients with short telomeres (less than the 10th percentile of normal subjects) are estimated from Refs. 9, 13, 25-27. 
"aging" of the lung, may underlie the mechanism of IPF due to telomerase mutations.

The central role of short telomere lengths in IPF has been demonstrated in two independent studies $(27,32)$. In one study, approximately $40 \%$ of individuals with FPF and $25 \%$ of sporadic cases have telomere lengths below the 10th percentile of normal control subjects (27). After separating out the individuals with coding mutations in telomerase (TERT and TERC), $24 \%$ of FPF kindreds and $23 \%$ of individuals with sporadic IIP have telomere lengths below the 10th percentile. This result is statistically significant, even after controlling for age, sex, and ethnicity. Thus, short telomeres may be a biological marker of telomerase dysfunction that identifies individuals who are susceptible to pulmonary fibrosis. Because the telomere lengths have been measured from circulating leukocytes, it is likely to reflect overall telomerase activity in bone marrow progenitor cells. It is intriguing to speculate that mutations in other genes that affect telomerase activity may be present in these IPF cases with very short telomere lengths. Because it is known that telomere lengths progressively shorten with advancing age, these findings provide a biologic explanation for the clinical observation of the age-related increased prevalence of IPF.

\section{TRANSLATION OF GENETIC RESULTS INTO CLINICAL PRACTICE}

Unlike cystic fibrosis, the genetics of IPF are complex. There is considerable genetic heterogeneity; rare mutations in four genes (TERT, TERC, SFTPC, and SFTPA2) have been identified so far (Figure 1). What effect will these discoveries have on patient care? First, all mutations found to date are very rare; the frequency of the SP-A2 mutations is less than $0.05 \%$ in an ethnicity-matched cohort. Although individually rare, these mutations have a large effect in the kindreds in which they are found. Inheritance of these rare heterozygous mutations segregates with pulmonary fibrosis in a Mendelian autosomaldominant pattern with reduced penetrance. One genome-wide association study has also identified a common variant in the TERT gene that is associated with IPF (33). The finding of both rare and common variants in the TERT gene provides compelling evidence of its involvement in the pathogenesis of IPF.

Second, the genetics of rare variants reveal biologic insights that are applicable to the more common sporadic form of disease. As discussed previously here, telomere shortening in the absence of germ line mutations in telomerase is seen in $25 \%$ of individuals with sporadic IIP. These findings have revealed a previously unknown underlying mechanism for pulmonary fibrosis. Similarly, the pathogenic mechanism of both the SP-C and SP-A2 mutations relates to their induction of ER stress in the alveolar type II cells. Korfei and colleagues (34) have shown a strong association between ER stress and alveolar type II cell apoptosis in sporadic IPF lung specimens. Again we see that the pathway identified by the study of rare mutations is relevant to the biology of common IPF. Drugs that prevent telomerase dysfunction in the distal lung or abate ER stress in the alveolar epithelium may show promise as novel therapeutics in the future.

Third, the identification of these genetic mutations will allow for the prediction of disease in susceptible individuals. Each first-degree relative within kindreds with a rare coding mutation in the surfactant or telomerase genes has a $50 \%$ chance of inheriting the same mutation as the proband. For those who do have a germ line mutation in the TERT gene, penetrance of pulmonary fibrosis is not $100 \%$. Instead, the development of pulmonary fibrosis is related to age, gender, and environmental exposures. For these at-risk carriers, the modification of their respiratory environment must be aggressively modified to limit fibrogenic exposures at an early age. Reduction in alveolar epithelial damage may delay or reduce the severity of disease. For those who do not have germ line mutations in the surfactant or telomerase genes, risk may be related to other genetic, epigenetic, or environmental influences that affect pathogenesis or progression of pulmonary fibrosis. Future studies that measure the association of disease with quantitative measurements of telomerase dysfunction or ER stress may provide predictions that are relevant for the general population.

Author Disclosure: C.K.G. has no disclosures.

\section{References}

1. American Thoracic Society/European Respiratory Society. International multidisciplinary consensus classification of the idiopathic interstitial pneumonias. This joint statement of the American Thoracic Society (ATS), and the European Respiratory Society (ERS) was adopted by the ATS board of directors, June 2001 and by the ERS executive committee, June 2001. Am J Respir Crit Care Med. 2002;165: 277-304.

2. Coultas DB, Zumwalt RE, Black WC, Sobonya RE. The epidemiology of interstitial lung diseases. Am J Respir Crit Care Med. 1994;150: 967-972.

3. Olson AL, Swigris JJ, Lezotte DC, Norris JM, Wilson CG, Brown KK. Mortality from pulmonary fibrosis increased in the United States from 1992 to 2003. Am J Respir Crit Care Med. 2007;176:277-284.

4. Raghu G, Weycker D, Edelsberg J, Bradford WZ, Oster G. Incidence and prevalence of idiopathic pulmonary fibrosis. Am J Respir Crit Care Med. 2006;174:810-816.

5. Marshall RP, Puddicombe A, Cookson WO, Laurent GJ. Adult familial cryptogenic fibrosing alveolitis in the UK. Thorax. 2000;55:143-146.

6. Loyd JE. Pulmonary fibrosis in families. Am J Respir Cell Mol Biol. 2003;29(3 Suppl):S47-S50.

7. Lee HL, Ryu JH, Wittmer MH, Hartman TE, Lymp JF, Tazelaar HD, Limper AH. Familial idiopathic pulmonary fibrosis: clinical features and outcome. Chest. 2005;127:2034-2041.

8. Steele MP, Speer MC, Loyd JE, Brown KK, Herron A, Slifer SH, Burch LH, Wahidi MM, Phillips JA III, Sporn TA, et al. Clinical and pathologic features of familial interstitial pneumonia. Am J Respir Crit Care Med. 2005;172:1146-1152.

9. Thomas AQ, Lane K, Phillips J III, Prince M, Markin C, Speer M, Schwartz DA, Gaddipati R, Marney A, Johnson J, et al. Heterozygosity for a surfactant protein $\mathrm{c}$ gene mutation associated with usual interstitial pneumonitis and cellular nonspecific interstitial pneumonitis in one kindred. Am J Respir Crit Care Med. 2002;165:1322-1328.

10. Nogee LM, Dunbar AE III, Wert SE, Askin F, Hamvas A, Whitsett JA. A mutation in the surfactant protein $\mathrm{c}$ gene associated with familial interstitial lung disease. N Engl J Med. 2001;344:573-579.

11. Bridges JP, Wert SE, Nogee LM, Weaver TE. Expression of a human surfactant protein $\mathrm{C}$ mutation associated with interstitial lung disease disrupts lung development in transgenic mice. J Biol Chem. 2003;278: 52739-52746.

12. Wang WJ, Mulugeta S, Russo SJ, Beers MF. Deletion of exon 4 from human surfactant protein $\mathrm{C}$ results in aggresome formation and generation of a dominant negative. J Cell Sci. 2003;116:683-692.

13. Lawson WE, Grant SW, Ambrosini V, Womble KE, Dawson EP, Lane $\mathrm{KB}$, Markin C, Renzoni E, Lympany $\mathrm{P}$, Thomas AQ, et al. Genetic mutations in surfactant protein $\mathrm{C}$ are a rare cause of sporadic cases of ipf. Thorax. 2004;59:977-980.

14. Wang Y, Kuan PJ, Xing C, Cronkhite JT, Torres F, Rosenblatt RL, Dimaio JM, Kinch LN, Grishin NV, Garcia CK. Genetic defects in surfactant protein A2 are associated with pulmonary fibrosis and lung cancer. Am J Hum Genet. 2009;84:52-59.

15. Maitra M, Wang Y, Gerard RD, Mendelson CR, Garcia CK. Surfactant protein A2 mutations associated with pulmonary fibrosis lead to protein instability and endoplasmic reticulum stress. $J$ Biol Chem. 2010;285:22103-22113.

16. Greider CW, Blackburn EH. Identification of a specific telomere terminal transferase activity in tetrahymena extracts. Cell. 1985;43:405-413.

17. Feng J, Funk WD, Wang SS, Weinrich SL, Avilion AA, Chiu CP, Adams RR, Chang E, Allsopp RC, Yu J, et al. The RNA component of human telomerase. Science. 1995;269:1236-1241.

18. Broccoli D, Young JW, de Lange T. Telomerase activity in normal and malignant hematopoietic cells. Proc Natl Acad Sci USA. 1995;92: 9082-9086. 
19. Wright WE, Piatyszek MA, Rainey WE, Byrd W, Shay JW. Telomerase activity in human germline and embryonic tissues and cells. Dev Genet. 1996;18:173-179.

20. Kim NW, Piatyszek MA, Prowse KR, Harley CB, West MD, Ho PL, Coviello GM, Wright WE, Weinrich SL, Shay JW. Specific association of human telomerase activity with immortal cells and cancer. Science. 1994;266:2011-2015.

21. Hastie ND, Dempster M, Dunlop MG, Thompson AM, Green DK, Allshire RC. Telomere reduction in human colorectal carcinoma and with ageing. Nature. 1990;346:866-868.

22. Calado RT, Young NS. Telomere disease. N Engl J Med. 2009;361:2353-2365.

23. Armanios M, Chen JL, Chang YP, Brodsky RA, Hawkins A, Griffin CA, Eshleman JR, Cohen AR, Chakravarti A, Hamosh A, et al. Haploinsufficiency of telomerase reverse transcriptase leads to anticipation in autosomal dominant dyskeratosis congenita. Proc Natl Acad Sci USA. 2005;102:15960-15964.

24. Armanios MY, Chen JJ, Cogan JD, Alder JK, Ingersoll RG, Markin C, Lawson WE, Xie M, Vulto I, Phillips JA III, et al. Telomerase mutations in families with idiopathic pulmonary fibrosis. $N$ Engl J Med. 2007;356: 1317-1326.

25. Tsakiri KD, Cronkhite JT, Kuan PJ, Xing C, Raghu G, Weissler JC, Rosenblatt RL, Shay JW, Garcia CK. Adult-onset pulmonary fibrosis caused by mutations in telomerase. Proc Natl Acad Sci USA. 2007; 104:7552-7557.

26. Diaz de Leon A, Cronkhite JT, Katzenstein AL, Godwin JD, Raghu G, Glazer CS, Rosenblatt RL, Girod CE, Garrity ER, Xing C, et al. Telomere lengths, pulmonary fibrosis and telomerase (TERT) mutations. PLoS ONE. 2010;5:e10680.
27. Cronkhite JT, Xing C, Raghu G, Chin KM, Torres F, Rosenblatt RL, Garcia CK. Telomere shortening in familial and sporadic pulmonary fibrosis. Am J Respir Crit Care Med. 2008;178:729-737.

28. Yamaguchi H, Calado RT, Ly H, Kajigaya S, Baerlocher GM, Chanock SJ, Lansdorp PM, Young NS. Mutations in TERT, the gene for telomerase reverse transcriptase, in aplastic anemia. $N$ Engl J Med. 2005;352:1413-1424.

29. Dokal I. Dyskeratosis congenita in all its forms. Br J Haematol. 2000;110: 768-779.

30. Garcia CK, Wright WE, Shay JW. Human diseases of telomerase dysfunction: Insights into tissue aging. Nucleic acids research. 2007;35: 7406-7416.

31. Selman M, Pardo A. Idiopathic pulmonary fibrosis: an epithelial/ fibroblastic cross-talk disorder. Respir Res. 2002;3:3.

32. Alder JK, Chen JJ, Lancaster L, Danoff S, Su SC, Cogan JD, Vulto I, Xie M, Qi X, Tuder RM, et al. Short telomeres are a risk factor for idiopathic pulmonary fibrosis. Proc Natl Acad Sci USA. 2008;105: 13051-13056.

33. Mushiroda T, Wattanapokayakit S, Takahashi A, Nukiwa T, Kudoh S, Ogura T, Taniguchi H, Kubo M, Kamatani N, Nakamura Y. A genome-wide association study identifies an association of a common variant in tert with susceptibility to idiopathic pulmonary fibrosis. J Med Genet. 2008;45:654-656.

34. Korfei M, Ruppert C, Mahavadi P, Henneke I, Markart P, Koch M, Lang G, Fink L, Bohle RM, Seeger W, et al. Epithelial endoplasmic reticulum stress and apoptosis in sporadic idiopathic pulmonary fibrosis. Am J Respir Crit Care Med. 2008;178:838-846. 